\title{
Model of a magnetic field in poloidal divertor tokamaks affected by resonant magnetic perturbations
}

S. S. Abdullaev

Citation: Physics of Plasmas 16, 030701 (2009); doi: 10.1063/1.3083293

View online: https://doi.org/10.1063/1.3083293

View Table of Contents: http://aip.scitation.org/toc/php/16/3

Published by the American Institute of Physics

\section{Articles you may be interested in}

Asymptotical and mapping methods in study of ergodic divertor magnetic field in a toroidal system Physics of Plasmas 6, 153 (1999); 10.1063/1.873270

On description of magnetic stochasticity in poloidal divertor tokamaks

Physics of Plasmas 15, 042508 (2008); 10.1063/1.2907163

Striation pattern of target particle and heat fluxes in three dimensional simulations for DIII-D

Physics of Plasmas 21, 020702 (2014); 10.1063/1.4864624

Modeling of stochastic magnetic flux loss from the edge of a poloidally diverted tokamak

Physics of Plasmas 9, 4957 (2002); 10.1063/1.1521125

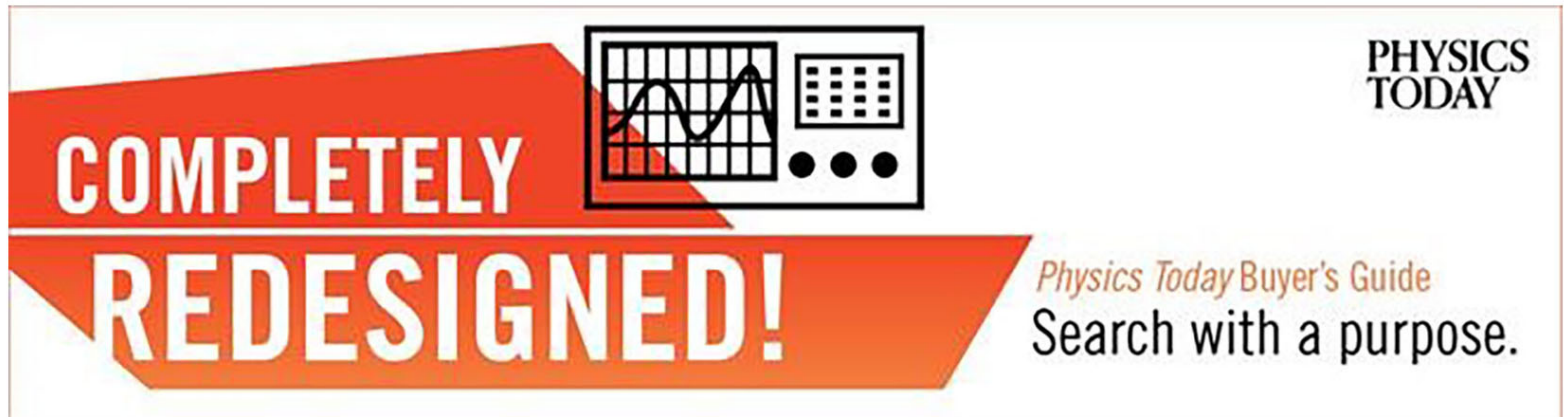




\title{
Model of a magnetic field in poloidal divertor tokamaks affected by resonant magnetic perturbations
}

\author{
S. S. Abdullaev \\ Forschungszentrum Jülich GmbH, Institute of Energy Research IEF-4: Plasma Physics, \\ Association EURATOM-FZJ, Partner in the Trilateral Euregio Cluster, 52425 Jülich, Germany
}

(Received 15 October 2008; accepted 27 January 2009; published online 3 March 2009)

A generic analytical model for the description of magnetic field lines in poloidal divertor tokamaks in the presence of external resonant magnetic perturbations is proposed. It is based on the Hamiltonian description of magnetic field lines in tokamaks. The safety factor and the spectra of magnetic perturbations are chosen by the requirement to satisfy their generic behavior near the magnetic separatrix and at the magnetic axis. The field line equations of the model are integrated using symplectic efficient mappings of field lines. The analytical formulas for the quasilinear diffusion and convection coefficients of field lines are obtained. The latter describes the outwardly directed transport of field lines at the plasma edge. It was shown that they are in a good agreement with the corresponding numerically calculated coefficients. () 2009 American Institute of Physics.

[DOI: $10.1063 / 1.3083293]$

The study of transport of energy and particles in stochastic fusion plasmas became an important issue in the light of recent experiments on the mitigation of large edge-localized modes (ELMs) in high-confinement $(H$-mode) plasmas by applied external resonant magnetic perturbations (RMPs) performed in tokamaks, Doublet III-D (DIII-D) and Joint European Torus (JET) (see, for example, Refs. 1-5). The possible use of RMPs field to suppress the ELMs in the future International Thermonuclear Experimental Reactor has a paramount importance for the protection of wall materials from the damaging effect of huge energy and particles releasing during ELMs pulses in the $H$-mode operation.

At the present time the mechanism of the ELM's suppression by the RMPs is not well understood (see Ref. 4). The first important step in this complex nonlinear problem of plasma dynamics is to study the structure and properties of the stochastic magnetic field at the plasma edge. For this purpose it would be useful to have a generic model of magnetic field near the magnetic separatrix of poloidal divertor tokamaks. Similar to the standard map for chaotic dynamical systems (see Refs. 6 and 7) such a model would allow fast and rigorous study of the structure and statistical properties of stochastic field lines at the plasma edge in the presence of RMPs. This model should be as simple as possible, and at the same time it should capture essential generic features of the magnetic field of the equilibrium plasma and RMPs near the magnetic separatrix.

In the present work we propose a generic analytical model for a magnetic field in poloidal divertor tokamaks affected by the external RMPs. The model is based on the Hamiltonian formulation of the equations for magnetic field lines and the new advancements in the description of Hamiltonian systems near the separatrix developed in Refs. 8 and 9 (see also Ref. 10).

Consider a magnetic field in a toroidal system in the presence of nonaxisymmetric magnetic perturbations. Suppose that the poloidal magnetic field has a first order null at the $X$ line (or $X$ point). Such a system is equivalent to a one-degree-of-freedom Hamiltonian system with a hyper- bolic fixed point subjected to a time-periodic perturbation. We use the Clebsch representation of the magnetic field $\mathbf{B}$ $=\nabla \psi_{t} \times \nabla \vartheta-\nabla \psi_{p} \times \nabla \varphi$ with the toroidal, $\psi_{t}$, and the poloidal, $\psi_{p}$, fluxes directed along the toroidal and poloidal directions, respectively, $\vartheta$ is the poloidal angle, and $\varphi$ is the toroidal angle. The equation of field lines in these variables has the Hamiltonian form

$$
\frac{d \vartheta}{d \varphi}=\frac{\partial \psi_{\mathrm{pol}}}{\partial \psi_{t}}, \quad \frac{d \psi_{t}}{d \varphi}=-\frac{\partial \psi_{\mathrm{pol}}}{\partial \vartheta}
$$

with $\vartheta$ and $\psi_{t}$ as canonically conjugated variables and $\psi_{\mathrm{pol}}$ as a Hamiltonian function. The latter in the presence of nonaxisymmetric magnetic perturbations can be presented as

$$
\psi_{p}\left(\vartheta, \psi_{t}, \varphi\right)=\int \frac{d \psi_{t}}{q\left(\psi_{t}\right)}+\psi_{p}^{(1)}\left(\vartheta, \psi_{t}, \varphi\right)
$$

where the first term in Eq. (2) represents the equilibrium plasma determined by the safety factor $q(\psi)$ and the second term, $\psi_{p}^{(1)}\left(\vartheta, \psi_{t}, \varphi\right)$, describes the magnetic perturbations. Further we use the toroidal flux $\psi \equiv \psi_{t}$ normalized to its maximum value at the separatrix, that is, the toroidal flux $0<\psi<1$ in the plasma region. The value $\psi=0$ corresponds to the magnetic axis, while $\psi=1$ corresponds to the magnetic separatrix.

The important element of the model is a presentation of the perturbation Hamiltonian $\psi_{p}^{(1)}$ describing the nonaxisymmetric perturbations in the presence of the RMPs. Let $n$ be a fundamental toroidal mode number. Then the general form of $\psi_{p}^{(1)}$ can be written as

$$
\begin{aligned}
& \psi_{p}^{(1)}(\vartheta, \psi, \varphi)=\sum_{s=1}^{\infty} \epsilon_{s} \psi_{s n}(\vartheta, \psi) \cos (\operatorname{sn} \varphi), \\
& \psi_{n}(\vartheta, \psi)=\sum_{m=1}^{\infty}\left|H_{m, n}(\psi)\right| \cos \left(m \vartheta+\chi_{m n}\right) .
\end{aligned}
$$


In Eq. (3) the quantities $H_{m n}(\psi)=\left|H_{m n}(\psi)\right| e^{i \chi_{m n}}$ describe the radial profiles of the poloidal modes $m$, and $\chi_{m n}$ are the corresponding phases. The perturbation equation (3) contains the $s n$ harmonics of the toroidal mode $n$. The dimensionless parameter $\epsilon_{s}$ stands for the perturbation strength of the $s$ th harmonics. Further we get $\epsilon_{s}=\epsilon / s$ and $\chi_{s n}=\chi_{n}$ for all toroidal modes $s n,(s=1,2, \ldots)$. This assumption agrees with the toroidal mode distribution of the perturbation field created by the rectangular current loops (see Ref. 11).

It is known that the magnetic perturbations near the separatrix have a broad spectrum in $m$. In order to find the asymptotics of $H_{m n}(\psi)$ for large $m$ we can use the relation between the generalized Poincaré integral $R_{n}(\psi)$ (see Refs. 9, 10 , and 12) and $H_{m n}(\psi)$ at the resonant magnetic surfaces $\psi_{m n}\left[q\left(\psi_{m n}\right)=m / n\right]$ :

$$
\left|R_{n}(\psi)\right|=2 \pi q(\psi)\left|H_{m n}(\psi)\right|=2 \pi(m / n)\left|H_{m n}(\psi)\right| .
$$

At the limit $\psi \rightarrow 1$ the function $R_{n}(\psi)$ has a finite value $R_{n}(1) \neq 0$, with the form $R_{n}(1) \sim \exp \left(-C_{0} n / \gamma\right)$, where $C_{0}$ is a constant independent of $n$ and $\gamma$ is a parameter in the asymptotics of the safety factor $q$ near the separatrix, i.e., $q(\psi)$ $\rightarrow(2 \pi \gamma)^{-1} \ln (Q /|h|)$ at $|h| \rightarrow 0$. Here $h \equiv h(\psi)=\psi_{p}(\psi)-\psi_{p}^{(s)}$ is the relative poloidal flux, $\psi_{p}^{(s)}=\psi_{p}(1)$ is the poloidal flux at the separatrix, and $Q$ is a constant. Such a dependence of $R_{n}$ on the toroidal mode number $n$ has been confirmed by numerous numerical and analytical calculations (see Refs. 9-11). According to the relation given by Eq. (4) the simplest form of the perturbation spectrum $H_{m, n}(\psi)$ which gives such an asymptotics of $R_{n}$ is

$$
H_{m n}(\psi)=\frac{n}{m} \exp \left(-m \frac{C(\psi)}{\gamma q(\psi)}\right),
$$

where $C(\psi)$ is an analytical function of $\psi$ with a finite value $C_{0}$ at $\psi=1$. Near the separatrix $\psi \rightarrow 1$ (or $|h| \rightarrow 0$ ) the amplitudes $H_{m n}(\psi)$ have the asymptotics $H_{m n}(\psi)$ $\sim \exp \left[-2 \pi m C_{0} / \ln (Q /|h|)\right]$. The latter shows the widening of the poloidal mode spectra when one approaches the separatrix.

Supposing that the phases $\chi_{m n}$ are independent of the poloidal mode number $m$, i.e., $\chi_{m n}=\chi_{n}$, one obtains the analytical formula for the perturbation function $\psi_{n}(\vartheta, \psi)$,

$$
\psi_{n}(\vartheta, \psi)=\psi_{n}^{(c)}(\vartheta, \psi) \cos \chi_{n}+\psi_{n}^{(s)}(\vartheta, \psi) \sin \chi_{n},
$$

where

$$
\begin{aligned}
& \psi_{n}^{(c)}(\vartheta, \psi)=-\frac{n}{2} \ln \Lambda, \quad \Lambda=1+e^{-2 \alpha}-2 e^{-\alpha} \cos \vartheta \\
& \psi_{n}^{(s)}(\vartheta, \psi)=n \arctan \left(\frac{\sin \vartheta}{\cos \vartheta-e^{\alpha}}\right), \quad \alpha=\frac{C(\psi)}{\gamma q(\psi)} .
\end{aligned}
$$

We choose the function $C(\psi)$ in the form $C(\psi)=C_{0} \psi$ $-\gamma q(\psi) \ln \psi / 2$, which gives a plausible behavior of the perturbation field inside the plasma, $H_{m n}(\psi) \propto \psi^{m / 2}$ at $\psi \ll 1$. The function $C(\psi)$ can be also established from the code calculations.

For the specific plasma the safety factor $q(\psi)$ can be

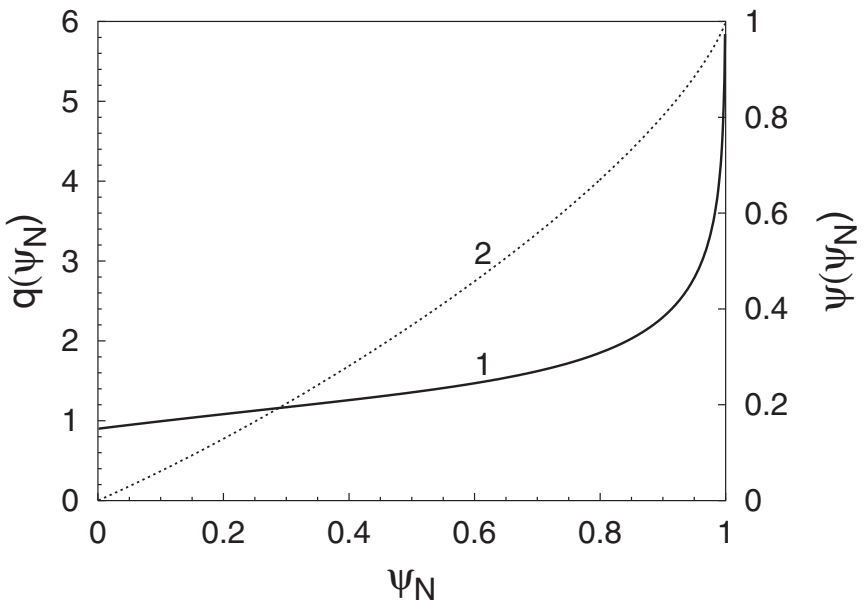

FIG. 1. Safety factor $q\left(\psi_{N}\right)$ given by Eq. (8) (curve 1) and the relation $\psi\left(\psi_{N}\right)$ given by Eq. (9) (curve 2) as functions of the normalized poloidal flux $\psi_{N}$ $=\left(h-h_{m}\right) /\left|h_{m}\right|$. Parameters: $\gamma=0.2, Q=1, h_{m}=-0.65$, and $q_{0}=0.9$. The value of $q_{95}=2.79$.

found from the equilibrium code calculations. For our model we use the following analytical presentation:

$$
q(\psi)=\frac{1}{2 \pi \gamma} \ln \frac{Q}{|h|}+a_{1}|h|+a_{2} h^{2},
$$

describing the well-known logarithmic asymptotics of $q(\psi)$ near the separatrix, $|h| \rightarrow 0$. In Eq. (8) the parameters $\gamma, Q$, $a_{1}$, and $a_{2}$ are independent of $h$, and they can be found from the equilibrium calculations or from the experiment.

From Eq. (8) one can find the relation between the toroidal flux $\psi$ and the relative poloidal flux $h$,

$$
\psi=1-\frac{|h|}{2 \pi \gamma}\left(\ln \frac{Q}{|h|}+1\right)-\frac{a_{1}}{2}|h|^{2}-\frac{a_{2}}{3}|h|^{3} .
$$

Instead of the parameters $a_{1}$ and $a_{2}$ we will choose the values $h_{m}$ and $q_{0}$ of $h$ and $q(\psi)$ at the magnetic axis of the plasma $\psi=0$, respectively, as free parameters.

Figure 1 shows the dependencies of the safety factor $q$ and the toroidal flux $\psi$ on the normalized poloidal flux $\psi_{N}$ $=\left(h-h_{m}\right) /\left|h_{m}\right|$. The angular dependence of the perturbation function (6) at several magnetic surfaces $\psi$ for the toroidal mode $n=3$ is shown in Fig. 2.

The Hamiltonian field line equations (1)-(3) can be effectively integrated using the mapping methods (see Ref. 10). We construct the mapping of field lines describing the evolution of canonical variables, the poloidal angle $\vartheta$ and the toroidal flux $\psi$ along the toroidal direction with the step along the toroidal angle equal to $2 \pi / n$.

Let $\left(\vartheta_{k}, \psi_{k}\right)$ be the values of canonical variables $(\vartheta, \psi)$ at the sections $\varphi_{k}=k 2 \pi / n(k=1,2, \ldots)$. We intend to construct the mapping describing the evolution of variables $\left(\vartheta_{k}, \psi_{k}\right)$ along the toroidal direction, i.e., $\left(\vartheta_{k}, \psi_{k}\right) \rightarrow\left(\vartheta_{k+1}, \psi_{k+1}\right)$. The general symplectic form of the mapping obtained by the method of canonical transformation of variables has the following form (see, e.g., Ref. 10): 


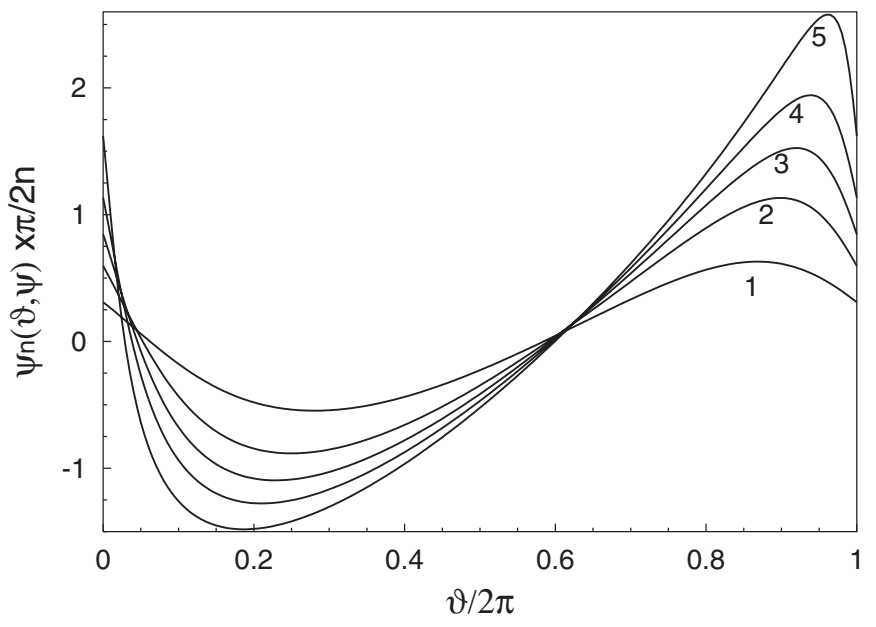

FIG. 2. Angular dependence of the perturbation function $\psi_{n}(\vartheta, \psi)$ given by Eq. (6) for the toroidal mode $n=3$ at the different values of $\psi: 1-\psi=0.1$, $2-\psi=0.3,3-\psi=0.5,4-\psi=0.7$, and $5-\psi=0.9$. The values of $C_{0}=0.07$ and the phase $\chi_{n}=1$ and other parameters are the same in Fig. 1.

$$
\begin{aligned}
& \Psi_{k}=\psi_{k}-\epsilon \frac{\partial S_{k}}{\partial \vartheta_{k}}, \quad \Theta_{k}=\vartheta_{k}+\epsilon \frac{\partial S_{k}}{\partial \Psi_{k}}, \\
& \bar{\Theta}_{k}=\Theta_{k}+\frac{\varphi_{k+1}-\varphi_{k}}{q\left(\Psi_{k}\right)}, \quad \Psi_{k+1}=\Psi_{k}, \\
& \psi_{k+1}=\Psi_{k+1}+\epsilon \frac{\partial S_{k+1}}{\partial \vartheta_{k+1}}, \quad \vartheta_{k+1}=\bar{\Theta}_{k}-\epsilon \frac{\partial S_{k+1}}{\partial \Psi_{k+1}},
\end{aligned}
$$

where $S_{k}$ and $S_{k+1}$ are values of the generating function $S$ $=S(\vartheta, \Psi, \varphi, \epsilon)$ at $\varphi=\varphi_{k}$ and $\varphi=\varphi_{k+1}$, respectively.

For the perturbation Hamiltonians (3) and (6) the generating function can be significantly simplified. One can show that in this case the generating functions $S_{k}$ and $S_{k+1}$ of the mapping are reduced to $S_{k}=S\left(\vartheta_{k}, \Psi_{k}\right)$ and $S_{k+1}$ $=-S\left(\vartheta_{k+1}, \Psi_{k+1}\right)$, respectively, where the function $S(\vartheta, \psi)$ $=\pi \psi_{n}(\vartheta, \Psi) / 2 n$ (see Ref. 10).

Figure 3 shows the mapping of field lines in the $(\vartheta, \psi)$ plane at the fixed poloidal sections $\varphi=\varphi_{k}$ obtained by the mapping given by Eq. (10).

To study the transport of particles and heat in the stochastic magnetic field one requires the knowledge of the diffusion coefficients of field lines and the Kolmogorov lengths characterizing the average length of exponential divergence of field lines (see, e.g., Ref. 13). As was shown in Ref. 12 the quasilinear diffusion coefficients of field lines in the stochastic layer closely describe ones obtained from the direct numerical calculations.

Using the quasilinear approximation for our analytical model one can obtain analytical formulas for the quasilinear diffusion coefficients. It is given by

$$
\begin{aligned}
& D_{\psi}^{(q)}(\psi)=D_{\psi}^{(q 0)} /(1-y), \\
& D_{\psi}^{(q 0)}(\psi)=(1 / 2) \pi n^{2} \epsilon^{2} q(\psi) y,
\end{aligned}
$$

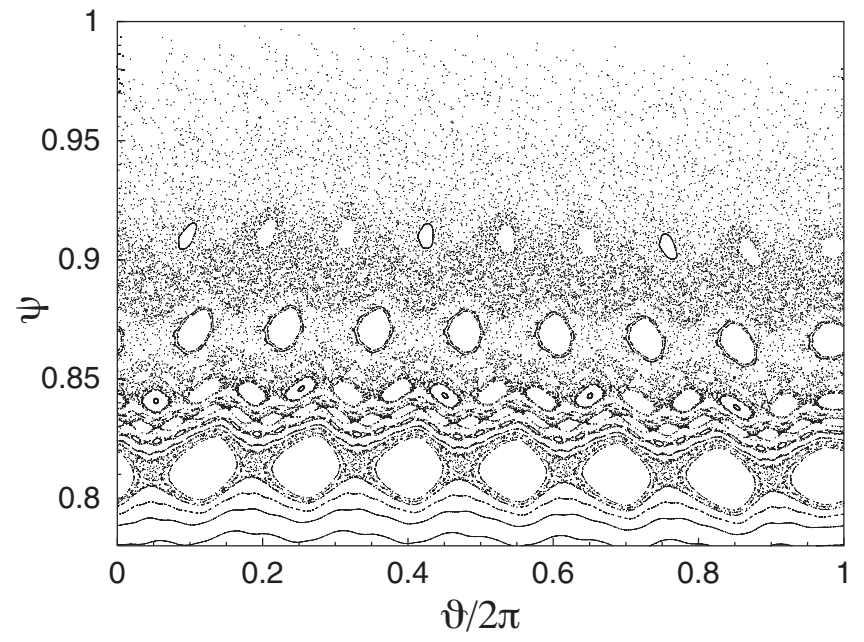

FIG. 3. Poincaré sections of field lines in the $(\vartheta, \psi)$ plane at the fixed sections $\varphi=(2 \pi / n) k$. The fundamental toroidal mode number $n=3$, the perturbation parameter $\epsilon=10^{-3}$, and the phase $\chi=1.0$.

where $y=\exp (-2 C(\psi) n / \gamma)$. The diffusion coefficient described by Eq. (12) corresponds to the perturbation equation (3) with the single toroidal mode $n$.

The profiles of field line diffusion coefficients $D_{\psi}(\psi)$ along the $\psi$ variable are plotted in Fig. 4: curves 1-3 correspond to the perturbation parameter $\epsilon=5 \times 10^{-4}$ and curves 4-6 correspond to $\epsilon=10^{-3}$. As seen from Fig. 4 the numerically found diffusion coefficients $D_{\psi}$ closely follow the corresponding quasilinear values $D_{\psi}^{(q)}(\psi)$ given by Eq. (11) but take slightly higher values than the quasilinear values. The difference between the quasilinear $D_{\psi}^{(q)}(\psi)$ and the numerical diffusion coefficients grows with increasing the perturbation amplitude.

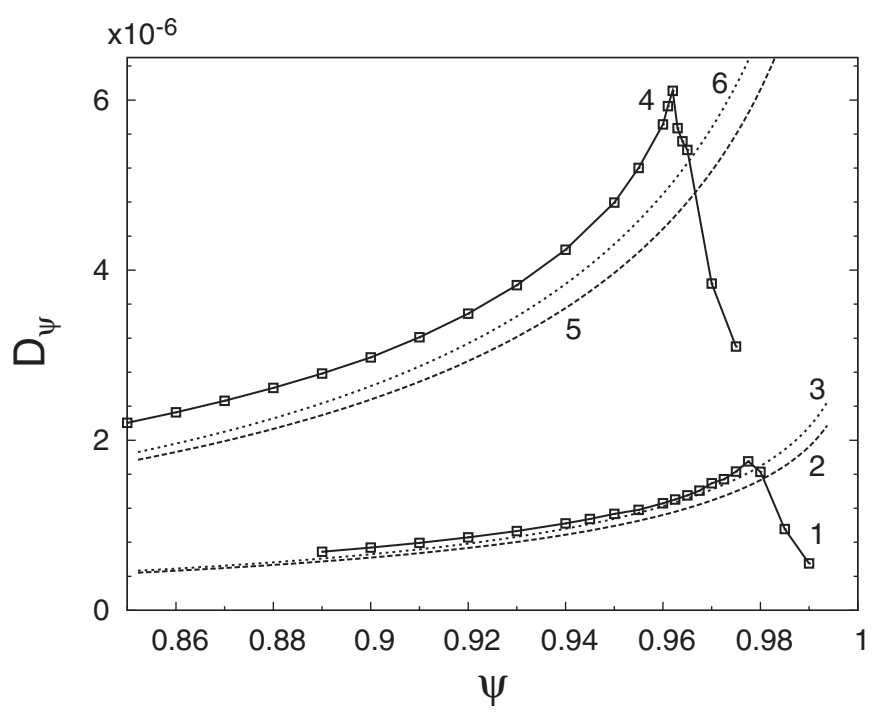

FIG. 4. Radial profiles of local field line diffusion coefficients $D_{\psi}$ calculated by the mapping (curves $1,2,5$, and 6). The corresponding quasilinear formula for the single toroidal mode perturbation $(s=1)$ given by Eq. (12) is described by curves 2 and 5 , and that for the infinity number of toroidal mode perturbations [Eq. (11)] is described by curves 3 and 6. Fundamental toroidal mode $n=3$ and perturbation parameters $\epsilon=5 \times 10^{-4}$ (curves 1-3) and $\epsilon=10^{-3}$ (curves 4-6). 
One can show that the diffusion coefficient $D_{\mathrm{FL}}$ $=\left\langle(\Delta X)^{2}\right\rangle / 2 \Delta l$ along the direction $X$ normal to the magnetic surface is $D_{\mathrm{FL}}=F^{-1} D_{\psi}$, where the factor $F$ is defined by the relation between $X$ and $\psi$. For the plasma with the minor radius $a=1 \mathrm{~m}$ and the major radius $R_{0}=3 \mathrm{~m}$ the factor $F$ monotonically changes from the value of 1 at $\psi=0.85$ to 0.74 at $\psi=0.96$. Thus the value of $D_{\mathrm{FL}}$ at $\psi=0.96$ reaches 8.4 $\times 10^{-6} \mathrm{~m}^{2} / \mathrm{m}$.

In Ref. 12 the field line convection coefficients characterizing the convectional transport of particles and heat due to chaotic field lines at the plasma edge have been introduced. It is defined as $u_{\psi}=\langle\Delta \psi\rangle / \Delta \varphi$, where $\langle\cdots\rangle$ stands for the averaging over field lines started on the given magnetic surface $\psi$. The quantity $u_{\psi}$ describes the average outward drift of field lines along the toroidal flux coordinate $\psi$ per unit toroidal angle $\Delta \varphi$. The nature of this outwardly directed transport is due to the radial dependence of the perturbation field and the quantity $u_{\psi}$ is related to the diffusion coefficient $D_{\psi}(\psi)$, i.e., $u_{\psi}(\psi)=d D_{\psi}(\psi) / d \psi$.

The corresponding coefficient $u_{\mathrm{FL}}=\langle\Delta X\rangle / \Delta l$ along the coordinate $X$ determines the convection (or pinch) velocity $V_{\perp}=v_{s} u_{\mathrm{FL}}$, where $v_{s}$ is the sound velocity.

The relation between $u_{\psi}$ and $u_{\mathrm{FL}}$ can be found to be similar to the one with the diffusion coefficients $D_{\psi}$ and $D_{\mathrm{FL}}$. Near the separatrix it is given by $u_{\mathrm{FL}}=F_{0}^{-1} u_{\psi}$, where the factor $F_{0}$ monotonically changes from 3.2 at $\psi=0.85$ to 1.2 at $\psi=0.98$.

The numerically calculated radial profiles of $u_{\psi}$ and their quasilinear estimations obtained from Eq. (11) are plotted in Fig. 5 for the two values of the perturbation parameter: $\epsilon$ $=5 \times 10^{-4}$ (curves 1 and 3 ) and $\epsilon=10^{-3}$ (curves 2 and 4).

As seen from Fig. 5 the coefficients $u_{\psi}$ closely follow their quasilinear estimations. They sharply grow when one approaches the unperturbed separatrix, i.e., at $\psi \rightarrow 1$.

The contribution of field \# lines to the convectional transport of particles and heat becomes of order of an anomalous convection velocity. For example, for the perturbation parameter $\epsilon=5 \times 10^{-4}$ the convection velocity $V_{\perp}=v_{s} u_{\mathrm{FL}}$ reaches the value of $18 \mathrm{~m} / \mathrm{s}$ at $\psi=0.98$ (for the sound velocity $v_{s}=5.5 \times 10^{5} \mathrm{~m} / \mathrm{s}$ ).

In summary we have proposed the simple analytical model of a magnetic field describing its generic properties near the magnetic separatrix of poloidal divertor tokamaks in the presence of external RMPs. The model allows fast study of the structure of magnetic field and estimations of statistical properties. The analytical expressions are found for the quasilinear field line diffusion coefficients. The latter are in a good agreement with the numerically calculated ones using the mapping. The mapping of field lines is employed to calculate the field line convection coefficients recently introduced to describe the average radial outward drift of open chaotic field lines. The model of magnetic field can be easily extended to the region outside the unperturbed separatrix. The latter would allow one to study the structure of magnetic

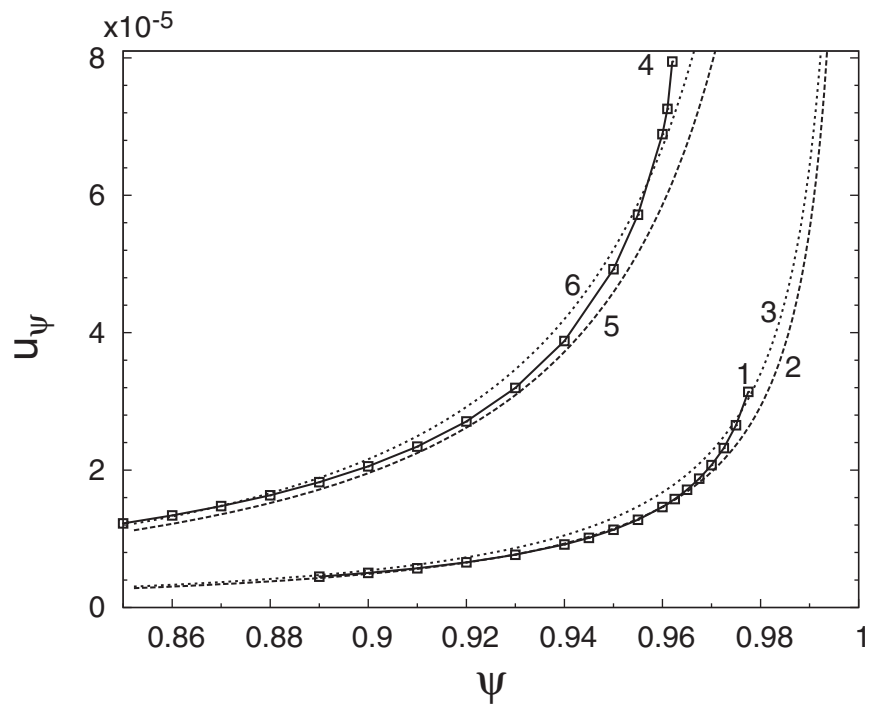

FIG. 5. Radial profiles of field line convection coefficients $u_{\psi}$ calculated numerically (curves 1 and 2) and their quasilinear estimations (curves 3-6). The parameters and the correspondence of curves are the same as in Fig. 4.

field outside the plasma region, particularly, magnetic footprints on the divertor plates.

The author acknowledges useful discussions with K. H. Finken, O. Schmitz, and M. Tokar.

${ }^{1}$ T. E. Evans, R. A. Moyer, P. R. Thomas, J. G. Watkins, T. H. Osborne, J. A. Boedo, E. J. Doyle, M. E. Fenstermacher, K. H. Finken, R. J. Groebner, M. Groth, J. H. Harris, R. J. LaHaye, C. J. Lasnier, S. Masuzaki, N. Ohyabu, D. G. Pretty, T. L. Rhodes, H. Reimerdes, D. L. Rudakov, M. J. Schaffer, G. Wang, and L. Zeng, Phys. Rev. Lett. 92, 235003 (2004).

${ }^{2}$ T. E. Evans, R. A. Moyer, J. G. Watkins, T. H. Osborne, P. R. Thomas, M. Becoulet, J. A. Boedo, E. J. Doyle, M. E. Fenstermacher, K. H. Finken, R. J. Groebner, M. Groth, J. H. Harris, G. L. Jackson, R. J. La Haye, C. J. Lasnier, S. Masuzaki, N. Ohyabu, D. G. Pretty, H. Reimerdes, T. L. Rhodes, D. L. Rudakov, M. J. Schaffer, M. R. Wade, G. Wang, W. P. West, and L. Zeng, Nucl. Fusion 45, 595 (2005).

${ }^{3}$ R. A. Moyer, T. Evans, T. H. Osborne, P. R. Thomas, M. Becoulet, J. Harris, K. H. Finken, J. A. Boedo, E. J. Doyle, M. E. Fenstermacher, P. Gohil, R. J. Groebner, M. Groth, G. L. Jackson, R. J. La Haye, C. J. Lasnier, A. W. Leonard, G. R. McKee, H. Reimerdes, T. L. Rhodes, D. L. Rudakov, M. J. Schaffer, P. B. Snyder, M. R. Wade, G. Wang, J. G. Watkins, W. P. West, and L. Zeng, Phys. Plasmas 12, 056119 (2005).

${ }^{4}$ T. E. Evans, R. A. Moyer, K. H. Burrel, M. E. Fenstermacher, I. Joseph, A. W. Leonard, T. H. Osborne, G. D. Porter, M. J. Schaffer, P. Snyder, P. Thomas, J. G. Watkins, and W. P. West, Nat. Phys. 2, 419 (2006).

${ }^{5}$ Y. Liang, H. R. Koslowski, P. R. Thomas, E. Nardon, B. Alper, P. Andrew, Y. Andrew, G. Arnoux, Y. Baranov, M. Bécoulet, M. Beurskens, T. Biewer, M. Bigi, K. Crombe, E. De La Luna, P. de Vries, W. Fundamenski, S. Gerasimov, C. Giroud, M. P. Gryaznevich, N. Hawkes, S. Hotchin, D. Howell, S. Jachmich, V. Kiptily, L. Moreira, V. Parail, S. D. Pinches, E. Rachlew, and O. Zimmerman, Phys. Rev. Lett. 98, 265004 (2007).

${ }^{6}$ B. V. Chirikov, Phys. Rep. 52, 263 (1979).

${ }^{7}$ A. J. Lichtenberg and M. A. Lieberman, Regular and Stochastic Motion (Springer, New York, 1992).

${ }^{8}$ S. S. Abdullaev, Phys. Rev. E 70, 046202 (2004).

${ }^{9}$ S. S. Abdullaev, Phys. Rev. E 72, 046202 (2005).

${ }^{10}$ S. S. Abdullaev, Construction of Mappings for Hamiltonian Systems and Their Applications, Lecture Notes in Physics Vol. 691 (Springer-Verlag, Berlin, 2006).

${ }^{11}$ S. S. Abdullaev, K. H. Finken, M. Jakubowski, and M. Lehnen, Nucl. Fusion 46, S113 (2006)

${ }^{12}$ S. S. Abdullaev, M. Jakubowski, M. Lehnen, O. Schmitz, and B. Unterberg, Phys. Plasmas 15, 042508 (2008).

${ }^{13}$ M. Z. Tokar, T. E. Evans, A. Gupta, R. Singh, P. Kaw, and R. C. Wolf, Phys. Rev. Lett. 98, 095001 (2007). 